\title{
PHYSICS OF INTENSE CHARGED PARTICLE BEAMS IN HIGH ENERGY ACCELERATORS
}


This page is intentionally left blank 


\section{PHYSICS OF INTENSE CHARGED PARTICLE BEAMS IN}

HIGH ENERGY ACCELERATORS

\section{Ronald C Davidson Hong Qin}

Plasma Physics Laboratory and

Department of Astrophysical Sciences

Princeton University 
Published by

Imperial College Press

57 Shelton Street

Covent Garden

London WC2H 9HE

and

World Scientific Publishing Co. Pte. Ltd.

P O Box 128, Farrer Road, Singapore 912805

USA office: Suite 1B, 1060 Main Street, River Edge, NJ 07661

UK office: 57 Shelton Street, Covent Garden, London WC2H 9HE

British Library Cataloguing-in-Publication Data

A catalogue record for this book is available from the British Library.

\section{PHYSICS OF INTENSE CHARGED PARTICLE BEAMS IN HIGH ENERGY ACCELERATORS}

Copyright $@ 2001$ by Imperial College Press and World Scientific Publishing Co. Pte. Ltd.

All rights reserved. This book, or parts thereof, may not be reproduced in any form or by any means, electronic or mechanical, including photocopying, recording or any information storage and retrieval system now known or to be invented, without written permission from the Publisher.

For photocopying of material in this volume, please pay a copying fee through the Copyright Clearance Center, Inc., 222 Rosewood Drive, Danvers, MA 01923, USA. In this case permission to photocopy is not required from the publisher.

ISBN 1-86094-300-4

ISBN 1-86094-301-2 (pbk)

Printed in Singapore by Uto-Print 


\section{FOR OUR CHILDREN}

\section{CYNTHIA, RONALD, JR., AND ANGELICA}

In deep appreciation for the joy and love they so generously share with family and friends. 
This page is intentionally left blank 


\section{Preface}

Periodic focusing and cyclic accelerators, transport systems and storage rings have a wide range of applications ranging from basic scientific research in high energy and nuclear physics, to applications such as spallation neutron sources, heavy ion fusion, tritium production, and nuclear waste treatment. Of particular importance, at the high beam currents and charge densities of practical interest, are the effects of the intense self fields produced by the beam space charge and current on determining the detailed equilibrium, stability and transport properties, and the nonlinear dynamics of the system. Intense charged particle beams (or charge bunches), like one-component nonneutral plasmas, are a many-body collection of charged particles which exhibit a broad range of collective phenomena, such as plasma waves and instabilities. Moreover, the intense self fields in a charged particle beam can have a large influence on the detailed dynamics and stability behavior of the beam.

During the past decade, the common physics of one-component nonneutral plasmas and intense charged particle beams has become increasingly evident. Indeed, many important scientific advances have been made, both in terms of theoretical understanding and instrumentation techniques, since the properties of intense charged particle beams and laboratory-confined nonneutral plasmas were investigated using similar theoretical formalisms in Physics of Nonneutral Plasmas (Addison Wesley, 1990; reissued by World Scientific, 2001). Of course an intense charged particle beam (or charge bunch), when considered in the beam frame, is in fact a one-component nonneutral plasma, which would be expected to exhibit many collective properties similar to laboratory-confined pure ion plasmas or pure elec- 
tron plasmas. In this regard, there is an extensive literature on the collective properties and nonlinear dynamics of laboratory-confined nonneutral plasmas which is relevant to the general subject matter of this book.

Physics of Intense Charged Particle Beams in High Energy Accelerators has been prepared as a graduate-level text which covers a broad range of topics related to the fundamental properties of the collective processes and nonlinear dynamics of intense charged particle beams. The subject matter is treated systematically from first principles using a unified theoretical approach, and the emphasis is on the development of basic concepts that illustrate the underlying physical processes in circumstances where intense self fields play a major role in determining the evolution of the system. The statistical models used to describe the properties of intense charged particle beams are based on the Vlasov-Maxwell equations, the macroscopic fluid-Maxwell equations, or the Klimontovich-Maxwell equations, as appropriate, and extensive use is made of theoretical techniques developed in the description of one-component nonneutral plasmas, and multispecies electrically-neutral plasmas, as well as established techniques in accelerator physics, classical mechanics, electrodynamics and statistical physics. These approaches are found to provide remarkably tractable and robust theoretical frameworks for describing the detailed nonlinear dynamics and collective processes in intense charged particle beams, including regimes where the intense self fields produced by the beam space charge and current play a major role in determining the detailed evolution of the system.

Physics of Intense Charged Particle Beams in High Energy Accelerators includes 75 problems and 71 figures and illustrations. Because of the book's emphasis on basic physics principles, and the thorough presentation format, it is intended to have a broad and lasting appeal to graduate students and researchers in the field.

Ronald C. Davidson Hong Qin

Princeton, New Jersey October, 2001 


\section{Acknowledgments}

The material for this book has evolved over more than one decade of teaching and research on the physics of intense charged particle beams and nonneutral plasmas. Many colleagues, students and collaborators in the accelerator physics and plasma physics communities have contributed significantly to the subject matter and selection of topics covered in this book. At the risk of overlooking key colleagues, we are particularly grateful for stimulating discussions with Roger Bangerter, John Barnard, Mike Blaskiewicz, Paul Channell, Alex Chao, Chiping Chen, Weiren Chou, Pat Colestock, Philip Efthimion, Nat Fisch, Alex Friedman, Irving Haber, Richard Hawryluk, Bill Herrmannsfeldt, Ingo Hofmann, Igor Kaganovich, Edward Lee, S. Y. Lee, Wei-li Lee, Grant Logan, Steve Lund, Robert Macek, Qian Qian, Martin Reiser, Gennady Shvets, Phillip Sprangle, Ed Startsev, Sean Strasburg, Stephan Tzenov, Han Uhm, Tai-Sen Wang, Tom Wangler, Jonathan Wurtele, Simon Yu, and Bruno Zotter.

Preparing the manuscript has been a significant technical undertaking. We are particularly indebted to Janet Wolfsheimer for her highly skilled typing of the manuscript and for her patience as we continued to add new material.

In addition, the preparation of the book has been possible only as a result of the encouragement and support of many individuals, institutions and government agencies over the years. In this regard, we particularly wish to thank Robert Goldston, Harold Shapiro, and Joseph Taylor of Princeton University; Ronald McKnight and John Willis of the United States Department of Energy, Office of Fusion Energy Sciences; Charles Roberson of the United States Office of Naval Research; and David Sutter of the United States Department 
of Energy, Division of High Energy Physics.

Finally, we would like to thank Jean Davidson and Heather Yu for their strong support and encouragement during the preparation of the material for this book.

\author{
Ronald C. Davidson \\ Hong Qin \\ Princeton, New Jersey
} October, 2001 


\section{Contents}

$\begin{array}{ll}\text { Preface } & \text { VII }\end{array}$

Acknowledgments $\quad$ IX

1 Introduction 1

1.1 Exordium . . . . . . . . . . . . . . 1

1.2 Historical Background . . . . . . . . . . . . 3

1.3 Characteristic Frequencies . . . . . . . . . . . 6

1.4 Outline of Contents . . . . . . . . . . . . 10

Chapter 1 References . . . . . . . . . . . . . . . . 12

2 Theoretical Models of Intense Nonneutral Particle Beams $\quad 23$

2.1 Introduction . . . . . . . . . . . . . . . . . 23

2.2 Vlasov-Maxwell Description . . . . . . . . . . . 26

2.3 Macroscopic Fluid Description _. . . . . . . . . 32

2.4 Conservation Relations . . . . . . . . . . . . . . 36

2.5 Discrete Particle Effects . . . . . . . . . . . . . . . 41

Chapter 2 References . . . . . . . . . . . . . . . . 46

3 Particle Orbits in Periodic Focusing Field Configurations $\quad 51$

3.1 Introduction . . . . . . . . . . . . . . 51

3.2 Periodic Focusing Field Configurations . . . . . . . . 54

3.3 Single-Particle Motion . . . . . . . . . . . . 66

3.3.1 Hamilton's Equations of Motion . . . . . . 67 
3.3.2 Orbit Equations in the Paraxial Approximation 69

3.3.3 Transverse Orbit Equations for a Continuous Beam ................ 75

3.4 Orbit and Envelope Equations for a Continuous Beam 79

3.4.1 Self-Field Potential for a Uniform Density Beam 79

3.4.2 Periodic Focusing Quadrupole Field . . . . . . 82

3.4.3 Periodic Focusing Solenoidal Field . . . . . . . 90

3.4.4 Uniform Focusing Field . . . . . . . . . . . . 98

3.5 Smooth Focusing Approximation . . . . . . . . . 103

3.6 Hamiltonian for Transverse Particle Motion . . . . . . 113

3.7 Matrix Transfer Formalism and Phase Advance . . . . 116

3.8 Orbit and Envelope Equations for a Finite-Length

Charge Bunch . . . . . . . . . . . . . . . . 128

Chapter 3 References . . . . . . . . . . . . . . . . . 140

4 Nonlinear Kinetic Stability Theorem 145

4.1 Introduction . . . . . . . . . . . . . . 145

4.2 Nonlinear Vlasov-Maxwell Equations in the Beam

Frame . . . . . . . . . . . . . . . . 148

4.3 Global Nonlinear Conservation Constraints . . . . . . 154

4.4 Three-Dimensional Kinetic Stability Theorem . . . . . 159

4.5 State of Minimum Helmholtz Free Energy . . . . . . 166

Chapter 4 References . . . . . . . . . . . . . . . . 173

5 Vlasov-Maxwell Description of Periodically-Focused Intense Beam Equilibria

5.1 Introduction . . . . . . . . . . . . . 177

5.1 .1 Theoretical Model . . . . . . . . . . . . . . 178

5.1.2 Focusing Field Configurations . . . . . . . . . . 181

5.2 Nonlinear Vlasov Equation and Hamilton's Equations of Motion . . . . . . . . . . . . . . . . . . . . 184

5.2.1 Conventional Hamiltonian Formulation . . . 185

5.2.2 Alternative Hamiltonian Formulation . . . . . . 189

5.3 Intense Beam Equilibria in a Uniform Focusing Field . 196

5.3.1 Isotropic Beam Equilibria . . . . . . . . . . 198 
5.3.2 Anisotropic Beam Equilibria . . . . . . . . . . 210

5.4 Intense Beam Equilibria in an Alternating-Gradient Quadrupole Field . . . . . . . . . . . . . . . . 222

5.4.1 Nonlinear Vlasov-Maxwell Equations . . . . . . 222

5.4.2 Periodically-Focused Beam Equilibria at Low Beam Intensity . . . . . . . . . . . . 224

5.4.3 Periodically-Focused Kapchinskij-Vladimirskij (KV) Intense Beam Equilibrium . . . . . . . . 234

5.5 Intense Beam Equilibria in a Periodic-Focusing Solenoidal Field . . . . . . . . . . . . . . . . . . 242

5.5.1 Nonlinear Vlasov-Maxwell Equations . . . . . . 242

5.5.2 Canonical Transformation to Larmor-Frame Variables . . . . . . . . . . . . . . . 244

5.5.3 Periodically-Focused Intense Beam Equilibria . 246 Chapter 5 References . . . . . . . . . . . . . . . . 253

6 Statistically-Averaged Rate Equations 257

6.1 Introduction and Theoretical Model . . . . . . . . 257

6.1.1 Alternating-Gradient Quadrupole Field . . . . 259

6.1.2 Periodic Focusing Solenoidal Field . . . . . . . 261

6.2 Rate Equations for Solenoidal Focusing Field . . . . . 263

6.2.1 General Rate Equation . . . . . . . . . . . . . 264

6.2.2 Conservation of Canonical Angular Momentum 265

6.2.3 Centroid Motion . . . . . . . . . . . . 267

6.2.4 Global Energy Balance . . . . . . . . . . . . 268

6.2.5 Rate Equation for Mean-Square Beam Radius . 271

6.2.6 Coupled Rate Equations for RMS Beam Radius and Transverse Emittance . . . . . . 273

6.3 Application of Rate Equations for a Solenoidal Focusing Field . . . . . . . . . . . 276

6.3.1 Rate Equations for Kapchinskij-Vladimirskij Beam Distribution . . . . . . . . . . . . 276

6.3.2 Rate Equations for Beam Distributions with Fixed-Shaped Density Profile . . . . . . . . . 279

6.4 Rate Equations for Quadrupole Focusing Field . . . . 283 
6.4.1 General Rate Equation . . . . . . . . . . . 284

6.4.2 Rate Equations for Average $x$ - and $y$ - Kinetic Energies . . . . . . . . . . . . 286

6.4.3 Rate Equations for Mean-Square Transverse Beam Dimensions . . . . . . . . . . . . 287

6.4.4 Coupled Rate Equations for Transverse Emittances and RMS Beam Dimensions . . . . 287

6.5 Rate Equations for Fixed-Shape Density Profile . . . . 290 Chapter 6 References . . . . . . . . . . . . . . . . . 296

7 Hamiltonian Averaging Techniques Applied to the Nonlinear Vlasov-Maxwell Equations 301

7.1 Introduction and Theoretical Model . . . . . . . . . 301

7.2 Canonical Transformation to Slow Variables . . . . 307

7.2.1 Canonical Transformation . . . . . . . . . . . 308

7.2.2 Third-Order Transformed Hamiltonian and Coordinate Transformation . . . . . . . . . . 319

7.3 Nonlinear Vlasov-Maxwell Equations in the Transformed Variables . . . . . . . . . . . . . . . . 323

7.3.1 Transformed Vlasov-Maxwell Equations . . . . 323

7.3.2 Equilibrium Properties $(\partial / \partial s=0)$ in the Transformed Variables . . . . . . . . . . 325

7.3.3 Density Inversion Theorem . . . . . . . . . . 331

7.3.4 Kinetic Stability Theorem . . . . . . . . . . . 332

7.3.5 Examples of Self-Consistent Beam Equilibria . 333

7.4 Periodically-Focused Beam Properties in the Laboratory Frame . . . . . . . . . . . . . . . 337

7.4.1 Laboratory-Frame Distribution Function . . . . 338

7.4.2 Statistical Averages in the Laboratory Frame . 340

7.4.3 Macroscopic Profiles in the Laboratory Frame . 343

7.4.4 Range of Validity of Asymptotic Expansion Procedure . . . . . . . . . . . 346

7.5 Conclusions . . . . . . . . . . . . . . 347

Chapter 7 References . . . . . . . . . . . . . . . 349 
8 Kinetic Stability Properties and Collective Oscillations in Intense Particle Beams

8.1 Introduction . . . . . . . . . . . . . 353

8.2 Assumptions and Theoretical Model . . . . . . . 355

8.3 Linearized Vlasov-Maxwell Equations and the Method of Characteristics . . . . . . . . . . . . . 358

8.4 Stability Properties for Perturbations About a Kapchinskij-Vladimirskij Beam Equilibrium . . . . . . 365

8.4.1 Eigenvalue Equation for $k_{z}=0 \ldots 365$

8.4.2 Stable Surface-Wave Oscillations for $\partial / \partial z=0$ and $\partial / \partial \theta \neq 0 \ldots \ldots \ldots \ldots \ldots \ldots$

8.4.3 Stability Properties for Axisymmetric Body-Wave Perturbations with $\partial / \partial z=0$ and $\partial / \partial \theta=0 \ldots \ldots \ldots \ldots \ldots \ldots$

8.5 Nonlinear Perturbative Particle Simulation Method for Describing Collective Processes . . . . . . . . . 383

8.6 Stability Properties for Perturbations about a Thermal Equilibrium Beam . . . . . . . . . . . . . . . . 389

8.6.1 Validation of Kinetic Stability Theorem . . . . 390

8.6.2 Normal-Mode Oscillations Determined from $\delta f$ Method for $\partial / \partial \theta=0=\partial / \partial z \ldots . . . .398$

Chapter 8 References . . . . . . . . . . . . . . . . . 401

9 Warm-Fluid Stability Properties and Collective Oscillations in Intense Particle Beams 407

9.1 Introduction . . . . . . . . . . . . . . . . . 407

9.2 Assumptions and Macroscopic Warm-Fluid Model . . 409

9.3 Macroscopic Equilibrium Properties . . . . . . . . . . 414

9.3.1 Equilibrium Force Balance . . . . . . . . . . . . 414

9.3.2 Warm-Fluid Thermal Equilibrium . . . . . . . 415

9.3.3 Warm-Fluid Kapchinskij-Vladimirskij (KV) Equilibrium . . . . . . . . . . . 4 416

9.3.4 Warm-Fluid Waterbag Equilibrium . . . . . . 418

9.3.5 Cold-Fluid Equilibrium . . . . . . . . . . 420

9.4 Linearized Fluid-Maxwell Equations . . . . . . . . . . 421 
9.5 Cold-Beam Eigenvalue Equation and Collective Oscillations . . . . . . . . . . . . . . . . . 423

9.6 Warm-Beam Stability Properties for Axisymmetric Flute Perturbations with $\partial / \partial \theta=0=\partial / \partial z \ldots 432$

9.6.1 Warm-Beam Eigenvalue Equation . . . . . . 432

9.6.2 Stability Properties for Perturbations about a Warm-Beam Kapchinskij-Vladimirskij Equilibrium . . . . . . . . . . . . 435

9.6.3 Stability Properties for Perturbations about the Warm-Beam Waterbag Equilibrium . . . . 440

9.7 Collective Oscillations and Instability Driven by Pressure Anisotropy . . . . . . . . . . . . . . . . . . . 448

9.7.1 Eigenvalue Equation for Perturbations about a Warm-Fluid Waterbag Equilibrium . . . . . . . 448

9.7.2 Stability Properties for $\hat{T}_{\| b}=0$ and $\partial / \partial \theta=0.451$ Chapter 9 References . . . . . . . . . . . . . . . 460

10 Special Topics on Intense Beam Propagation 465

10.1 Introduction . . . . . . . . . . . . . . . 465

10.2 Intense Beam Propagation Through Background Plasma . . . . . . . . . . . . . . . . . 466

10.2.1 Self-Pinched Beam Propagation - The Bennett Pinch . . . . . . . . . . 466

10.2.2 Alfvén-Lawson Limiting Current . . . . . . . 470

10.2.3 The Plasma Lens Effect . . . . . . . . . . . . 473

10.3 Collisionless Damping (Growth) of Collective

Excitations . . . . . . . . . . . . 478

10.3.1 The Landau Dielectric Function . . . . . . . 479

10.3.2 Kinetic Dispersion Relation for a Multi-component Plasma . . . . . . . . . . 485

10.3.3 Landau Damping of Electron Plasma Oscillations . . . . . . . . . . . . 495

10.3.4 Collisionless Damping of Dipole-Mode

Sideband Oscillations in Intense Particle Beams 499 
10.4 Electron-Ion Two-Stream Instability in Intense

Particle Beams . . . . . . . . . . . . . . . . 503

10.4.1 Kinetic Dispersion Relation . . . . . . . . . 504

10.4.2 Nonlinear $\delta f$ Simulations of the

Electron-Proton Two-Stream Instability . . . . 515

10.5 Paul Trap Simulator of Intense Beam Propagation . . 522

10.6 Halo Particle Production by Collective Mode

Excitations . . . . . . . . . . . . . 531

10.7 Probing the Beam Microstate . . . . . . . . . . . 541

10.7.1 The Density Inversion Theorem . . . . . . . . 542

10.7.2 Collective Excitations . . . . . . . . . . . . 544

10.7.3 Beam Echoes . . . . . . . . . . . . . . 548

Chapter 10 References . . . . . . . . . . . . . . . 555

Supplementary References $\quad 563$

$\begin{array}{ll}\text { Subject Index } & 577\end{array}$ 